\title{
Limites hydrodynamiques pour les équations de Vlasov-Stokes
}

\section{Thierry Goudon Pierre-Emmanuel Jabin Alexis Vasseur \\ Résumé}

On présente quelques problèmes et résultats de type limites hydrodynamiques pour des modèles couplés fluide/cinétique décrivant l'interaction de particules avec un fluide en mouvement.

\section{Introduction}

On s'intéresse à la description d'un nuage de particules interagissant avec un fluide. Les particules sont décrites par l'intermédiaire d'une fonction de distribution : $f(t, x, v) \geq 0$ est la densité de particules qui, à l'instant $t \geq 0$, occupent la position $x \in \mathbb{R}^{3}$, avec la vitesse $v \in \mathbb{R}^{3}$ (On présente le modèle dans le cadre tri-dimensionnel, plus réaliste physiquement, bien que par la suite on soit amené à se restreindre à une situation 2D). Autrement dit, $\int_{\Omega} \int_{\mathcal{V}} f(t, x, v) \mathrm{d} x \mathrm{~d} v$ est le nombre de particules qui, à l'instant $t$, occupent le domaine $\Omega \times \mathcal{V}$ de l'espace des phases. Cette quantité obéit à une équation de type Vlasov

$$
\partial_{t} f+\nabla_{x} \cdot(v f)+\nabla_{v} \cdot(F f)=r \Delta_{v} f
$$

où $F$ représente la densité des forces exercées sur les particules. Le terme de droite décrit les effets d'agitation brownienne, avec $r>0$.

La densité du fluide $\rho_{g}$ est supposée constante (i.e. on néglige le volume occupé par les particules) et le fluide est décrit par l'évolution de son champ de vitesses $u(t, x) \in \mathbb{R}^{3}$ qui satisfait l'équation de Navier-Stokes incompressible

$$
\left\{\begin{array}{l}
\rho_{g}\left(\partial_{t} u+\operatorname{Div}_{x}(u \otimes u)+\nabla_{x} p\right)-\mu \Delta_{x} u=\mathfrak{F}, \\
\operatorname{div}_{x}(u)=0 .
\end{array}\right.
$$

Ici $\mu>0$ désigne la viscosité dynamique du fluide et $\mathfrak{F}$ représente la densité de forces exercées sur le fluide.

Le couplage entre ces deux équations est réalisé par les termes de force. Pour les décrire, on doit introduire les quantités suivantes :

Une part de l'activité de Th. Goudon est réalisée à l'INRIA Sophia, projet CAIMAN. MSC 2000 : 35Q99, 35B25.

Keywords : Vlasov-Stokes equations, Hydrodynamic limit, Fluid-particles interaction. 
- le rayon des particules $a>0$, supposé constant (il n'y a pas ici de phénomènes de coagulation ou fragmentation);

- les densités massiques $\rho_{p}, \rho_{g}$ des particules et du fluide, respectivement;

- la masse $M$ des particules, donnée par $M=\frac{4}{3} \pi \rho_{p} a^{3}$.

En négligeant la gravité, les forces exercées sur une particule se réduisent aux forces de traînée engendrées par le fluide (force de Stokes). Ces efforts sont proportionnels à la vitesse relative de la particule et du fluide

$$
F=\frac{6 \pi \mu a}{M}(u-v)=\frac{9 \mu}{2 a^{2} \rho_{p}}(u-v) .
$$

Par ailleurs, le coefficient de diffusion est déterminé par la formule d'Einstein

$$
r=\frac{k T}{M} \frac{6 \pi \mu a}{M}=\frac{k T}{M} \frac{9 \mu}{2 a^{2} \rho_{p}}
$$

qui fait intervenir la constante de Boltzmann $k$ et la température $T>0$ de la suspension, supposée constante. Enfin, l'ensemble des particules exerce sur le fluide une force donnée par

$$
\mathfrak{F}=-\rho_{p} \int_{\mathbb{R}^{3}} F f \mathrm{~d} v=\frac{9 \mu}{2 a^{2}} \int_{\mathbb{R}^{3}} f(v-u) \mathrm{d} v .
$$

En résumé, on s'intéresse donc au système d'EDP suivant

$$
\left\{\begin{array}{l}
\partial_{t} f+v \cdot \nabla_{x} f+\nabla_{v} \cdot\left(\frac{9 \mu}{2 a^{2} \rho_{p}}(u-v) f\right)=\frac{k T}{M} \frac{9 \mu}{2 a^{2} \rho_{p}} \Delta_{v} f \\
\rho_{g}\left(\partial_{t} u+\operatorname{Div}_{x}(u \otimes u)+\nabla_{x} p\right)-\mu \Delta_{x} u=\frac{9 \mu}{2 a^{2}} \int_{\mathbb{R}^{3}} f(v-u) \mathrm{d} v \\
\operatorname{div}_{x}(u)=0
\end{array}\right.
$$

complété de données initiales $f_{0}, u_{0}$.

Ecrivons maintenant ce système sous forme adimensionnelle. On introduit des unités de temps et de longueur, notées $\mathfrak{T}$ et $L$ respectivement. Dans les expressions précédentes, apparaissent le temps de relaxation

$$
\tau=\frac{M}{6 \pi \mu a}=\frac{2 a^{2} \rho_{p}}{9 \mu}
$$

et la vitesse thermique

$$
\sqrt{\theta}=\left(\frac{k T}{M}\right)^{1 / 2}
$$

On définit alors les variables sans dimension suivantes

$$
t=\mathfrak{T} \bar{t}, \quad x=L \bar{x}, \quad v=\sqrt{\theta} \bar{v}
$$

et les inconnues adimensionnelles

$$
\bar{f}(\bar{t}, \bar{x}, \bar{v})=\sqrt{\theta}^{3} f(\mathfrak{T} \bar{t}, L \bar{x}, \sqrt{\theta} \bar{v}), \quad u=\sqrt{\theta} \bar{u} .
$$


Alors (1) se réécrit

$$
\left\{\begin{array}{l}
\frac{1}{\mathfrak{T}} \partial_{\bar{t}} \bar{f}+\frac{\sqrt{\theta}}{L} \bar{v} \cdot \nabla_{\bar{x}} \bar{f}+\frac{1}{\sqrt{\theta}} \nabla_{\bar{v}} \cdot\left(\frac{\sqrt{\theta}}{\tau}(\bar{u}-\bar{v}) \bar{f}\right)=\frac{\theta}{\sqrt{\theta}^{2} \tau} \Delta_{\bar{v}} \bar{f}, \\
\frac{\sqrt{\theta}}{\mathfrak{T}} \partial_{\bar{t}} \bar{u}+\frac{\theta}{L} \operatorname{Div}_{x}(\bar{u} \otimes \bar{u})+\nabla_{x} \bar{p}-\frac{\sqrt{\theta} \mu}{\rho_{g} L^{2}} \Delta_{\bar{x}} \bar{u}=\frac{9 \mu}{2 a^{2}} \frac{1}{\rho_{g}} \sqrt{\theta} \int_{\mathbb{R}^{N}} \bar{f}(\bar{v}-\bar{u}) \mathrm{d} \bar{v} \\
\operatorname{div}_{x}(\bar{u})=0 .
\end{array}\right.
$$

On remarque que $\mu=M /(6 \pi \tau a)=\left(2 a^{2} \rho_{p}\right) /(9 \tau)$. Dans ces équations intervient donc le jeu de grandeurs physiques sans dimension suivant

$$
\left\{\begin{aligned}
A & =\frac{\mathfrak{T}}{L} \sqrt{\theta}, & B & =\frac{\mathfrak{T}}{\tau}, \\
D & =\frac{\mathfrak{T}}{\tau} \frac{\rho_{p}}{\rho_{g}}=B \frac{\rho_{p}}{\rho_{g}}, & E & =\frac{9}{2}\left(\frac{a}{L}\right)^{2} \frac{\mathfrak{T}}{\tau} \frac{\rho_{p}}{\rho_{g}}=\frac{9}{2}\left(\frac{a}{L}\right)^{2} D .
\end{aligned}\right.
$$

En allégeant la notation, (2) prend la forme

$$
\left\{\begin{array}{l}
\partial_{t} f+A v \cdot \nabla_{x} f+B \nabla_{v} \cdot\left((u-v) f-\nabla_{v} f\right)=0 \\
\partial_{t} u+A \operatorname{Div}_{x}(u \otimes u)+\nabla_{x} p-E \Delta_{x} u=D \rho(V-u)=D(J / A-\rho u), \\
\operatorname{div}_{x}(u)=0
\end{array}\right.
$$

où on a noté

$$
\begin{aligned}
& \rho(t, x)=\int_{\mathbb{R}^{N}} f(t, x, v) \mathrm{d} v \quad \text { (densité macroscopique de particules), } \\
& \rho V(t, x)=\int_{\mathbb{R}^{N}} v f(t, x, v) \mathrm{d} v=J(t, x) / A \quad \text { (vitesse macroscopique de particules). }
\end{aligned}
$$

Notons qu'on peut réécrire dans l'équation cinétique

$$
\nabla_{v} \cdot\left((u-v) f-\nabla_{v} f\right)=-\nabla_{v} \cdot\left(M_{u} \nabla_{v}\left(f M_{u}^{-1}\right)\right), \quad M_{u}(v)=e^{-|v-u|^{2} / 2} .
$$

Sous cette forme symétrique, on devine que l'équation a pour effet de relaxer vers la Maxwellienne de vitesse $u$. On va maintenant discuter les ordres de grandeur des quantités dans (3) en terme d'un paramètre $\varepsilon>0$, destiné à tendre vers 0 et traiter le problème de perturbation singulière correspondant.

Le modèle a été présenté de manière détaillée par Caflisch-Papanicolaou [3]; on trouvera aussi de nombreux détails dans [24] en relation avec des problèmes de combustion, ainsi que dans l'article récent de Desvillettes [5]. Des modèles de ce type sont décrits aussi dans Clouet-Domelevo [4], avec une approche probabiliste, ou dans Russo-Smerecka [22], Herrero-Lucquin-Perthame [15], Jabin-Perthame [18] pour des écoulements potentiels. L'analyse mathématique du système (1) a été menée à bien par Hamdache [13] (existence de solutions, comportement en temps grand,...) qui a aussi présenté certains problèmes de type limite hydrodynamique dans [14]. Ces questions ont été abordées, pour des problèmes légèrement différents, par Berthonnaud [1], Domelevo-Roquejoffre [7], Domelevo-Vignal [8], Goudon [12], 
Jabin $[16,17], \ldots$ Une des difficultés rencontrées est liée, lorsqu'il n'y a pas de terme de diffusion dans l'équation cinétique, à la possible apparition de phénomènes de concentration. Des difficultés analogues interviennent aussi en physique des plasmas ou en astrophysique comme étudié par Poupaud-Soler [21], Nieto-PoupaudSoler [20].

\section{Limite "parabolique"}

On s'intéresse au choix suivant dans (3)

$$
A=\varepsilon^{-1}, \quad B=\varepsilon^{-2}, \quad E=1=D,
$$

où le paramètre $\varepsilon$ est destiné à tendre vers 0 . Ceci signifie que la vitesse de référence $L / \mathfrak{T}$ est petite par rapport à la vitesse thermique (ordre $\varepsilon$ ) et le temps de relaxation est petit par rapport à l'échelle de temps d'observation $\mathfrak{T}$ (ordre $\varepsilon^{2}$ ). Notons aussi que dans ce choix, on a $\rho_{p} \sim \varepsilon^{2} \rho_{g}$ et $a \sim L$. Le système (4) devient

$$
\left\{\begin{array}{l}
\partial_{t} f^{\varepsilon}+\frac{1}{\varepsilon} v \cdot \nabla_{x} f^{\varepsilon}+\frac{1}{\varepsilon^{2}} \nabla_{v} \cdot\left(\left(u^{\varepsilon}-v\right) f^{\varepsilon}-\nabla_{v} f^{\varepsilon}\right)=0, \\
\partial_{t} u^{\varepsilon}+\frac{1}{\varepsilon} \operatorname{Div}_{x}\left(u^{\varepsilon} \otimes u^{\varepsilon}\right)+\nabla_{x} p^{\varepsilon}-\Delta_{x} u^{\varepsilon}=\rho^{\varepsilon}\left(V^{\varepsilon}-u^{\varepsilon}\right)=\left(\varepsilon J^{\varepsilon}-\rho^{\varepsilon} u^{\varepsilon}\right), \\
\operatorname{div}_{x}\left(u^{\varepsilon}\right)=0 \\
\rho^{\varepsilon}(t, x)=\int f^{\varepsilon}(t, x, v) \mathrm{d} v, \quad \rho^{\varepsilon} V^{\varepsilon}(t, x)=\int v f^{\varepsilon}(t, x, v) \mathrm{d} v=\varepsilon J^{\varepsilon}(t, x) .
\end{array}\right.
$$

\subsection{Estimations a priori}

On se place à partir de maintenant dans un cadre bi-dimensionel $x \in \mathbb{R}^{2}, v \in$ $\mathbb{R}^{2}$ (les formules peuvent changer, mais l'analyse dimensionnelle est analogue). On suppose que les données initiales $f_{0}^{\varepsilon} \geq 0, f_{0}^{\varepsilon} \in L^{1}\left(\mathbb{R}^{2} \times \mathbb{R}^{2}\right)$ et $u_{0}^{\varepsilon} \in L^{2}\left(\mathbb{R}^{2}\right)$ vérifient les estimations uniformes suivantes

$$
\left\{\begin{array}{l}
\int_{\mathbb{R}^{2}} \int_{\mathbb{R}^{2}} f_{0}^{\varepsilon}\left(1+x^{2}+v^{2}+\left|\ln \left(f_{0}^{\varepsilon}\right)\right|\right) \mathrm{d} v \mathrm{~d} x \leq C_{0}, \\
\int_{\mathbb{R}^{2}}\left|u_{0}^{\varepsilon}\right|^{2} \mathrm{~d} x \leq C_{0} \varepsilon^{2}
\end{array}\right.
$$

pour $C_{0}>0$, indépendant de $\varepsilon$. L'estimation cruciale est la suivante

Proposition 1 Soit $0<T<\infty$. On a

$$
\begin{aligned}
& \int_{\mathbb{R}^{2}} \int_{\mathbb{R}^{2}} f^{\varepsilon}(1\left.+x^{2}+v^{2}+\left|\ln \left(f^{\varepsilon}\right)\right|\right) \mathrm{d} v \mathrm{~d} x+\int_{\mathbb{R}^{2}}\left|\frac{u^{\varepsilon}}{\varepsilon}\right|^{2} \mathrm{~d} x \\
&+\frac{1}{\varepsilon^{2}} \int_{0}^{T} \int_{\mathbb{R}^{2}} \int_{\mathbb{R}^{2}}\left|\left(u^{\varepsilon}-v\right) \sqrt{f^{\varepsilon}}-2 \nabla_{v} \sqrt{f^{\varepsilon}}\right|^{2} \mathrm{~d} v \mathrm{~d} x \mathrm{~d} t \\
&+\int_{0}^{T} \int_{\mathbb{R}^{2}}\left|\frac{\nabla_{x} u^{\varepsilon}}{\varepsilon}\right|^{2} \mathrm{~d} x \mathrm{~d} t \leq C
\end{aligned}
$$

où $C$ ne dépend que de $T$ et de (5). 
Cet énoncé appelle quelques commentaires :

- Les données sont préparées en fonction du scaling choisi : le champ de vitesses initial tend vers 0 . En conséquence, la vitesse du fluide $u^{\varepsilon}$ tend vers 0 (en norme $L^{2}$ ). On travaillera donc sur la vitesse "rescalée" $\widetilde{u}^{\varepsilon}=\varepsilon^{-1} u^{\varepsilon}$. Ceci signifie que la vitesse du fluide est petite vis-à-vis de la vitesse thermique.

- Il est bien clair qu'une borne $L^{1}$ ne suffit pas à garantir une propriété de compacité $L^{1}$ faible comme l'illustrent les exemples classiques de $n \chi_{[0,1 / n]}(x)$ et $\chi_{[n, n+1](x)}$ sur $\mathbb{R} \ldots$ En revanche, une suite $h_{n}$ qui vérifie

$$
\int_{\mathbb{R}^{N}}\left|h_{n}\right|\left(1+\omega(x)+G\left(\left|h_{n}\right|\right)\right) \mathrm{d} x \leq C,
$$

avec $\lim _{|x| \rightarrow \infty} \omega(x)=\infty$ et $\lim _{s \rightarrow \infty} G(s) / s=\infty$ est faiblement compacte dans $L^{1}\left(\mathbb{R}^{N}\right)$. Le poids $\omega$ évite les fuites à l'infini, l'estimation avec la fonction $G$, "plus forte" que $L^{1}$, évite les concentrations. On dispose donc d'estimations de ce type, avec $\omega(x, v)=x^{2}+v^{2}, G(s)=s|\ln (s)|$. Ici, un autre intérêt de ce type d'estimation est que, en dimension 2 , on peut donner un sens au produit d'une fonction $L \ln L$ par une fonction $H^{1}$ (voir le Lemme 5).

- Le terme de dissipation $\frac{1}{\varepsilon}\left(\left(u^{\varepsilon}-v\right) \sqrt{f^{\varepsilon}}-2 \nabla_{v} \sqrt{f^{\varepsilon}}\right)$ peut se réécrire avec la Maxwellienne

$$
M^{\varepsilon}(t, x, v)=\exp \left(-\frac{\left|v-u^{\varepsilon}(t, x)\right|^{2}}{2}\right) .
$$

On a

$$
\frac{1}{\varepsilon}\left(\left(u^{\varepsilon}-v\right) \sqrt{f^{\varepsilon}}-2 \nabla_{v} \sqrt{f^{\varepsilon}}\right)=-\frac{2}{\varepsilon} \sqrt{M^{\varepsilon}} \nabla_{v} \sqrt{\frac{f^{\varepsilon}}{M^{\varepsilon}}} .
$$

En supposant formellement que $u^{\varepsilon} \rightarrow 0, f^{\varepsilon} \rightarrow f$ "suffisamment fortement", on est conduit à

$$
\left(u^{\varepsilon}-v\right) \sqrt{f^{\varepsilon}}-2 \nabla_{v} \sqrt{f^{\varepsilon}} \rightarrow 0=-v \sqrt{f}-2 \nabla_{v} \sqrt{f}
$$

ce qui signifie que la limite $f$ n'est rien d'autre que la Maxwellienne

$$
f(t, x, v)=\rho(t, x) e^{-v^{2} / 2}(2 \pi)^{-N / 2} .
$$

Le point clef de la preuve réside dans le calcul de la dérivée de l'énergie cinétique et de l'entropie associée à $f^{\varepsilon}$, combinée à l'énergie cinétique du fluide. Précisément, on a

$$
\begin{gathered}
\begin{array}{c}
\frac{d}{d t}\left(\int_{\mathbb{R}^{2}} \int_{\mathbb{R}^{2}} f^{\varepsilon}\left(v^{2}+\ln \left(f^{\varepsilon}\right)\right) \mathrm{d} v \mathrm{~d} x+\int_{\mathbb{R}^{2}}\left|\frac{u^{\varepsilon}}{2 \varepsilon}\right|^{2} \mathrm{~d} x\right)+\int_{\mathbb{R}^{2}}\left|\frac{\nabla_{x} u^{\varepsilon}}{2 \varepsilon}\right|^{2} \mathrm{~d} x \\
=\frac{1}{\varepsilon^{2}} \int_{\mathbb{R}^{2}} \int_{\mathbb{R}^{2}}\left(u^{\varepsilon}-v\right) f^{\varepsilon} \cdot\left(v+\frac{\nabla_{v} f^{\varepsilon}}{f^{\varepsilon}}\right) \mathrm{d} v \mathrm{~d} x+\frac{1}{\varepsilon^{2}} \int_{\mathbb{R}^{2}} \int_{\mathbb{R}^{2}}\left(v-u^{\varepsilon}\right) f^{\varepsilon} \cdot u^{\varepsilon} \mathrm{d} v \mathrm{~d} x \\
\quad-\frac{1}{\varepsilon^{2}} \int_{\mathbb{R}^{2}} \int_{\mathbb{R}^{2}} \frac{\left|\nabla_{v} f^{\varepsilon}\right|^{2}}{f^{\varepsilon}} \mathrm{d} v \mathrm{~d} x+\frac{1}{\varepsilon^{2}} \int_{\mathbb{R}^{2}} \int_{\mathbb{R}^{2}} v \cdot \nabla_{v} f^{\varepsilon} \mathrm{d} v \mathrm{~d} x \\
=-\frac{1}{\varepsilon^{2}} \int_{\mathbb{R}^{2}} \int_{\mathbb{R}^{2}}\left(\left|u^{\varepsilon}-v\right|^{2} f^{\varepsilon}+\frac{\mid \nabla_{v} f^{2}}{f^{\varepsilon}}-2\left(v-u^{\varepsilon}\right) \cdot \nabla_{v} f^{\varepsilon}\right) \mathrm{d} v \mathrm{~d} x \\
=-\frac{1}{\varepsilon^{2}} \int_{\mathbb{R}^{2}} \int_{\mathbb{R}^{2}}\left|\left(v-u^{\varepsilon}\right) f^{\varepsilon}-2 \nabla_{v} \sqrt{f^{\varepsilon}}\right|^{2} \mathrm{~d} v \mathrm{~d} x \\
=-\frac{1}{\varepsilon^{2}} \int_{\mathbb{R}^{2}} \int_{\mathbb{R}^{2}} M^{\varepsilon}\left|\nabla_{v}\left(f^{\varepsilon} / M^{\varepsilon}\right)\right|^{2} \mathrm{~d} v \mathrm{~d} x .
\end{array}
\end{gathered}
$$


On contrôle $\iint x^{2} f^{\varepsilon} \mathrm{d} v \mathrm{~d} x$ par l'entropie et l'énergie puisque

$$
\frac{d}{d t} \iint x^{2} f^{\varepsilon} \mathrm{d} v \mathrm{~d} x=\iint x \cdot v f^{\varepsilon} \mathrm{d} v \mathrm{~d} x \leq \iint\left(x^{2}+v^{2}\right) f^{\varepsilon} \mathrm{d} v \mathrm{~d} x .
$$

Enfin, on utilise des astuces classiques en théorie cinétique pour, à partir des informations sur $\iint\left(1+x^{2}+v^{2}+\ln \left(f^{\varepsilon}\right)\right) f^{\varepsilon} \mathrm{d} v \mathrm{~d} x$, contrôler la quantité positive $\iint(1+$ $\left.x^{2}+v^{2}+\left|\ln \left(f^{\varepsilon}\right)\right|\right) f^{\varepsilon} \mathrm{d} v \mathrm{~d} x$. De cette estimation concernant la quantité microscopique $f^{\varepsilon}$, on déduit des estimations sur les grandeurs macroscopiques

$$
\rho^{\varepsilon}(t, x)=\int_{\mathbb{R}^{2}} f^{\varepsilon} \mathrm{d} v, \quad J^{\varepsilon}=\frac{1}{\varepsilon} \int_{\mathbb{R}^{2}} v f^{\varepsilon} \mathrm{d} v .
$$

Lemme 1 Sous les hypothèses de la Proposition 1, $\rho^{\varepsilon}\left(1+x^{2}+\left|\ln \left(\rho^{\varepsilon}\right)\right|\right)$ est bornée dans $L^{\infty}\left(0, T ; L^{1}\left(\mathbb{R}^{2}\right)\right)$.

Cette estimation se déduit des propriétés de $f^{\varepsilon}$ par des techniques connues en équations cinétiques (voir par exemple, [19]).

Lemme 2 La quantité $J^{\varepsilon}-\rho^{\varepsilon} \widetilde{u}^{\varepsilon}=J^{\varepsilon}-\rho^{\varepsilon} u^{\varepsilon} / \varepsilon$ est bornée dans $L^{\infty}\left(0, T ; L^{1}\left(\mathbb{R}^{2}\right)\right)$.

On exploite la relation relation (7) en remarquant que

$$
J^{\varepsilon}-\rho^{\varepsilon} \widetilde{u}^{\varepsilon}=\frac{1}{\varepsilon} \int 2 \sqrt{M^{\varepsilon}} \nabla_{v}\left(\sqrt{f^{\varepsilon} / M^{\varepsilon}}\right) \sqrt{f^{\varepsilon}} \mathrm{d} v
$$

On fait ainsi apparaître le terme de dissipation et conclut à l'aide de l'inégalité de Cauchy-Schwarz.

Lemme 3 La quantité $\rho^{\varepsilon} V^{\varepsilon}=\varepsilon J^{\varepsilon}$ est bornée dans $L^{\infty}\left(0, T ; L^{1}\left(\mathbb{R}^{2}\right)\right)$. De plus, pour $i$ dans $\{1,2\},\left|\rho^{\varepsilon} V_{i}^{\varepsilon}\right| \sqrt{\left[\ln \left(\left|\rho^{\varepsilon} V_{i}^{\varepsilon}\right|\right)\right]_{+}}$est bornée dans $L^{\infty}\left(0, T ; L^{1}\left(\mathbb{R}^{2}\right)\right)$.

La première de ces estimations est une conséquence de la borne sur $\iint(1+$ $\left.v^{2}\right) f^{\varepsilon} \mathrm{d} v \mathrm{~d} x$. En fait, on va voir avec le Lemme 4 que cette quantité tend vers 0 dans $L_{\text {loc }}^{1}$. La seconde estimation sera utile sur le plan technique, elle peut s'obtenir en suivant par exemple les idées de [19].

Lemme 4 Soit $0<R<\infty$. Alors $\rho^{\varepsilon} \widetilde{u}^{\varepsilon}$ est borné dans $L^{2}\left(0, T ; L^{1}\left(B_{R}\right)\right)$ et le courant $J^{\varepsilon}$ est borné dans $L^{2}\left(0, T ; L^{1}\left(B_{R}\right)\right)$.

Le contrôle du courant se déduit du Lemme 2 et de l'estimation sur $\rho^{\varepsilon} u^{\varepsilon}$. Celle-ci exploite la restriction à la dimension 2 et les estimations (par rapport à la variable d'espace) $L^{1} \cap L \ln L$ sur $\rho^{\varepsilon}$ et $H^{1}$ sur $u^{\varepsilon}$ : voir le Lemme 5 . 


\subsection{Analyse formelle}

Grâce aux bornes discutées plus haut, on peut supposer que

$$
\rho^{\varepsilon} \rightarrow \rho, \quad u^{\varepsilon} \rightarrow u, \quad J^{\varepsilon} \rightarrow J
$$

On considère les équations de moment. En multipliant l'équation de Vlasov par 1 et en intégrant en $v$, on obtient la relation de conservation

$$
\partial_{t} \rho^{\varepsilon}+\operatorname{div}_{x} J^{\varepsilon}=0
$$

Quand $\varepsilon$ tend vers 0 , ceci devient

$$
\partial_{t} \rho+\operatorname{div}_{x} J=0 .
$$

Ensuite, en multipliant l'équation de Vlasov par $v$ et en intégrant en $v$, on est conduit à

$$
\varepsilon^{2} \partial_{t} J^{\varepsilon}+\operatorname{Div}_{x} \mathbb{P}^{\varepsilon}=\frac{1}{\varepsilon} \int_{\mathbb{R}^{2}}\left(\left(u^{\varepsilon}-v\right) f^{\varepsilon}+\nabla_{v} f^{\varepsilon}\right) \mathrm{d} v=\rho^{\varepsilon} \widetilde{u}^{\varepsilon}-J^{\varepsilon},
$$

où le terme de pression cinétique est donné par les seconds moments de $f^{\varepsilon}$

$$
\mathbb{P}^{\varepsilon}=\int_{\mathbb{R}^{2}} v \otimes v f^{\varepsilon} \mathrm{d} v
$$

On réécrit $\mathbb{P}^{\varepsilon}$ de manière à faire intervenir le terme de dissipation. On a

$$
\begin{aligned}
\mathbb{P}^{\varepsilon}=\int_{\mathbb{R}^{2}}\left(\left(v-u^{\varepsilon}\right) \sqrt{f^{\varepsilon}}+2 \nabla_{v} \sqrt{f^{\varepsilon}}\right) \otimes v \sqrt{f^{\varepsilon}} \mathrm{d} v & \\
& +\int_{\mathbb{R}^{2}} u^{\varepsilon} \otimes v f^{\varepsilon} \mathrm{d} v-2 \int_{\mathbb{R}^{2}} \nabla_{v} \sqrt{f^{\varepsilon}} \otimes v \sqrt{f^{\varepsilon}} \mathrm{d} v .
\end{aligned}
$$

En utilisant l'inégalité de Cauchy-Schwarz et la Proposition 1, on remarque que la première intégrale est $\mathcal{O}(\varepsilon)$ (dans $\left.L^{1}\left((0, T) \times \mathbb{R}^{2}\right)\right)$. Le second terme dans (12) est en fait

$$
u^{\varepsilon} \otimes \rho^{\varepsilon} V^{\varepsilon}=u^{\varepsilon} \otimes \varepsilon J^{\varepsilon}=\varepsilon \widetilde{u}^{\varepsilon} \otimes \rho^{\varepsilon} V^{\varepsilon} .
$$

Il s'exprime sous la forme $\varepsilon \times$ (produit de quantités bornées); on peut donc s'attendre à ce que ce terme s'annule dans la limite $\varepsilon \rightarrow 0$. Finalement, le dernier terme n'est rien d'autre que $2 \rho^{\varepsilon}$. Si le produit $\rho^{\varepsilon} \widetilde{u}^{\varepsilon}$ passe à la limite, on obtient donc en faisant tendre $\varepsilon$ vers 0 dans (11)

$$
2 \nabla_{x} \rho=\rho \tilde{u}-J .
$$

En insérant l'expression ainsi obtenue pour $J$ dans (10), on est conduit à l'équation de Kramers-Smoluchowski pour la densité $\rho$

$$
\partial_{t} \rho+\operatorname{div}_{x}\left(\rho \widetilde{u}-2 \nabla_{x} \rho\right)=0
$$

c'est-à-dire une équation de dérive-diffusion, de vitesse $\widetilde{u}$, de coefficient de diffusion 2 . 
Il reste à décrire l'équation satisfaite par $\widetilde{u}$. En divisant l'équation de NavierStokes par $\varepsilon$, on obtient l'équation suivante pour la vitesse rescalée

$$
\partial_{t} \widetilde{u}^{\varepsilon}+\operatorname{Div}_{x}\left(\widetilde{u}^{\varepsilon} \otimes \widetilde{u}^{\varepsilon}\right)-\Delta \widetilde{u}^{\varepsilon}+\nabla_{x} \widetilde{p}^{\varepsilon}=J^{\varepsilon}-\rho^{\varepsilon} \widetilde{u}^{\varepsilon},
$$

complétée par la relation d'incompressibilié $\operatorname{div}_{x} \widetilde{u}^{\varepsilon}=0$. D'après le Lemme 4 , le terme de droite de (13) est borné dans $L^{2}\left(0, T ; L^{1}\left(\mathbb{R}^{2}\right)\right)$. En fait, en revenant à (11), on peut le mettre sous la forme $J^{\varepsilon}-\rho^{\varepsilon} \widetilde{u}^{\varepsilon}=-\operatorname{Div}_{x}\left(\mathbb{P}^{\varepsilon}\right)+$ "petit" $\rightarrow-2 \nabla_{x} \rho$ qui peut s'incorporer dans un terme de pression. Ainsi, on supposant que la convergence de $\widetilde{u}^{\varepsilon}$ est assez forte pour passer à la limite dans le terme quadratique de convection, on obtient

$$
\partial_{t} \widetilde{u}+\operatorname{Div}_{x}(\widetilde{u} \otimes \widetilde{u})-\Delta \widetilde{u}+\nabla_{x} \widetilde{\pi}=0,
$$

i.e. $\widetilde{u}$ est solution de l'équation de Navier-Stokes incompressible. Pour justifier ce passage à la limite, les difficultés mathématiques sont donc :

a) justifier que le produit $\rho^{\varepsilon} V^{\varepsilon} \otimes \widetilde{u}^{\varepsilon}$ est borné,

b) justifier que le produit $\rho^{\varepsilon} \widetilde{u}^{\varepsilon}$ passe à la limite,

c) justifier que la convergence de $\widetilde{u}^{\varepsilon}$ est assez forte pour passer à la limite dans l'équation fluide.

\subsection{Passage à la limite}

Le point le plus simple est le point c. On va montrer que la suite $\widetilde{u}^{\varepsilon}$ est relativement compacte dans $L^{2}\left((0, T) \times B_{R}\right)$, pour tout $0<R<\infty$. D'après la Proposition $1, \widetilde{u}^{\varepsilon}$ est borné dans $L^{\infty}\left(0, T ; L^{2}\left(\mathbb{R}^{2}\right)\right) \cap L^{2}\left(0, T ; H^{1}\left(\mathbb{R}^{2}\right)\right)$. On va conclure en appliquant en argument de type "lemme d'Aubin" (voir par exemple [23]) qui s'appuie sur une estimation supplémentaire de $\partial_{t} \widetilde{u}^{\varepsilon}$ dans un espace de type $L^{r}$ en temps, à valeurs dans un Sobolev d'exposant négatif. Le seul terme qui reste à contrôler est le gradient de pression. Mais, en prenant la divergence de l'équation (13), on obtient

$$
\Delta \widetilde{p}^{\varepsilon}=\operatorname{div}_{x}\left(R^{\varepsilon}\right) \quad \text { avec } \quad R^{\varepsilon}(t, x)=\left(J^{\varepsilon}-\rho^{\varepsilon} \widetilde{u}^{\varepsilon}\right)-\left(\widetilde{u}^{\varepsilon} \cdot \nabla_{x}\right) \widetilde{u}^{\varepsilon} .
$$

D'après la Proposition $1, R^{\varepsilon}$ est bornée dans $L^{2}\left(0, T ; L^{1}\left(\mathbb{R}^{2}\right)\right)$. Or

$$
\begin{aligned}
\widetilde{p}^{\varepsilon}(t, x) & =\int_{\mathbb{R}^{2}} \frac{1}{2 \pi} \ln (|x-y|) \nabla_{x} \cdot R^{\varepsilon}(t, y) \mathrm{d} y \\
& =\int_{\mathbb{R}^{2}} \frac{x-y}{2 \pi|x-y|^{2}} \cdot R^{\varepsilon}(t, y) \mathrm{d} y=\int_{|x-y| \leq r} \ldots \mathrm{d} y+\int_{|x-y| \geq r} \ldots \mathrm{d} y
\end{aligned}
$$

On en déduit que $\widetilde{p}^{\varepsilon}$ est borné dans $L^{2}\left(0, T ; L^{1}+L^{\infty}\left(\mathbb{R}^{2}\right)\right)$. Il s'ensuit que $\partial_{t} \widetilde{u}^{\varepsilon}$ est bornée dans $L^{2}\left(0, T ; W^{-1,1}\left(B_{R}\right)\right)$.

Le point $b$ résulte du résultat général suivant.

Lemme 5 On considère deux suites de fonctions $a^{\varepsilon}, b^{\varepsilon}: \mathbb{R}^{2} \rightarrow \mathbb{R}$ vérifiant

$$
\sup _{t \in(0, T)} \int_{\mathbb{R}^{2}}\left|a^{\varepsilon}\right|\left(1+\sqrt{\left[\ln \left(\left|a^{\varepsilon}\right|\right)\right]_{+}}\right) \mathrm{d} x \leq C,
$$


et

$$
\sup _{t \in(0, T)} \int_{\mathbb{R}^{2}}\left|b^{\varepsilon}\right|^{2} \mathrm{~d} x \leq C, \quad \int_{0}^{T} \int_{\mathbb{R}^{2}}\left|\nabla_{x} b^{\varepsilon}\right|^{2} \mathrm{~d} x \mathrm{~d} t \leq C .
$$

Alors, pour tout $\varphi \in C_{c}^{\infty}\left(\mathbb{R}^{2}\right)$, la quantité $\int_{0}^{T}\left|\int_{\mathbb{R}^{2}} a^{\varepsilon} b^{\varepsilon} \varphi \mathrm{d} x\right|^{2} \mathrm{~d} s$ est bornée indépendemment de $\varepsilon$.

On applique ce résultat avec $a^{\varepsilon}=\rho^{\varepsilon} V^{\varepsilon}$ et $b^{\varepsilon}=\widetilde{u}^{\varepsilon}$. Ceci permet bien de se débarasser, dans le développement de la pression cinétique, de $\varepsilon \rho^{\varepsilon} V^{\varepsilon} \otimes \widetilde{u}^{\varepsilon}$ lorsque $\varepsilon \rightarrow 0$. On peut justifier cet énoncé de plusieurs façon ; l'une des plus simples consiste à exploiter l'inégalité de Trudinger, [10] (Th. 7.15, p. 162).

On pose $\beta^{\varepsilon}=b^{\varepsilon} \varphi$ qui est bornée dans $L^{\infty}\left(0, T ; L^{2}\left(\mathbb{R}^{2}\right)\right)$ et $L^{2}\left(0, T ; H^{1}\left(\mathbb{R}^{2}\right)\right)$, à support dans $(0, T) \times B_{R}$. On note $\left\|\nabla_{x} \beta^{\varepsilon}\right\|(t)=\left(\int_{\mathbb{R}^{2}}\left|\nabla_{x} \beta\right|^{2} \mathrm{~d} x\right)^{1 / 2} \in L^{2}(0, T)$. Il existe des constantes $\sigma, K>0$ telles que

$$
\sup _{t \in(0, T)} \int_{B_{R}} \exp \left(\sigma\left(\frac{\left|\beta^{\varepsilon}(t, x)\right|}{\left\|\nabla_{x} \beta^{\varepsilon}\right\|(t)}\right)^{2}\right) \mathrm{d} x \leq K
$$

Alors, il vient

$$
\begin{aligned}
& \int_{\mathbb{R}^{2}}\left|a^{\varepsilon} b^{\varepsilon} \varphi\right| \mathrm{d} x=\int_{\left|a^{\varepsilon}\right| \leq 1} \ldots \mathrm{d} x+\int_{1 \leq\left|a^{\varepsilon}\right| \leq \exp \left(\sigma|\beta|^{2} /\left(2\|\nabla \beta\|^{2}\right)\right)} \ldots \mathrm{d} x \\
& +\int_{\left|a^{\varepsilon}\right| \geq \exp \left(\sigma|\beta|^{2} /\left(2\|\nabla \beta\|^{2}\right)\right)} \cdots \mathrm{d} x \\
& \leq \int_{\mathbb{R}^{2}}\left|\beta^{\varepsilon}\right| \mathrm{d} x+\int_{\mathbb{R}^{2}}\left|\beta^{\varepsilon}\right| \exp \left(\sigma|\beta|^{2} /\left(2\|\nabla \beta\|^{2}\right)\right) \mathrm{d} x \\
& +\sqrt{2 / \sigma}\|\nabla \beta\| \int_{\mathbb{R}^{N}}\left|a^{\varepsilon}\right| \sqrt{\left[\ln \left(\left|a^{\varepsilon}\right|\right)\right]_{+}} \mathrm{d} x,
\end{aligned}
$$

qui permet de conclure à l'aide d'inégalités de Cauchy-Schwarz et en exploitant les estimations sur $a^{\varepsilon}, b^{\varepsilon}$.

Il ne reste plus qu'à montrer que le produit $\rho^{\varepsilon} u^{\varepsilon}$ passe à la limite (point a)). A cette fin, on s'appuie sur l'énoncé suivant, où l'on renforce les hypothèses sur la suite $a^{\varepsilon}$.

Proposition 2 On suppose que a $a^{\varepsilon}$ vérifie

$$
\sup _{t \in(0, T)} \int_{\mathbb{R}^{2}}\left|a^{\varepsilon}\right|\left(1+\left|\ln \left(\left|a^{\varepsilon}\right|\right)\right|\right) \mathrm{d} x \leq C,
$$

et

$$
\partial_{t} a^{\varepsilon} \text { est borné dans } L^{2}\left(0, T ; W_{\mathrm{loc}}^{-m, p}\left(\mathbb{R}^{2}\right)\right)
$$

pour $m \geq 0, p \geq 1$. On suppose que $b^{\varepsilon}$ vérifie (14). On suppose aussi que

$$
\begin{cases}a^{\varepsilon} \rightarrow a & \text { faiblement dans } L_{\mathrm{loc}}^{1}\left((0, T) \times \mathbb{R}^{2}\right), \\ b^{\varepsilon} \rightarrow b & \text { faiblement dans } L^{2}\left((0, T) \times \mathbb{R}^{2}\right), \\ \nabla_{x} b^{\varepsilon} \rightarrow \nabla_{x} b & \text { faiblement dans } L^{2}\left((0, T) \times \mathbb{R}^{2}\right) .\end{cases}
$$

Alors, le produit $a^{\varepsilon} b^{\varepsilon}$ passe à la limite

$$
a^{\varepsilon} b^{\varepsilon} \rightarrow a b \quad \text { in } \mathcal{D}^{\prime}\left((0, T) \times \mathbb{R}^{2}\right) .
$$


Moralement, dans cet énoncé on combine un gain de compacité en espace pour l'une des suites (borne sur $\nabla_{x} b^{\varepsilon}$ ) avec un gain de compacité en temps pour l'autre suite (borne sur $\partial_{t} a^{\varepsilon}$ ). On trouvera des énoncés de même esprit dans Dumas-Golse [9], Lions [19]. L'idée de la démonstration est la suivante. Soit $\varphi \in C_{c}^{\infty}\left((0, T) \times \mathbb{R}^{2}\right)$. On doit montrer que $\iint a^{\varepsilon} b^{\varepsilon} \phi \mathrm{d} x \mathrm{~d} t \rightarrow \iint a b \phi \mathrm{d} x \mathrm{~d} t$ quand $\varepsilon \rightarrow 0$. On écrit $\beta^{\varepsilon}=b^{\varepsilon} \phi$ en utilisant la solution élémentaire du laplacien $E(x)=\frac{1}{2 \pi} \ln (|x|)$ comme suit

$$
\beta^{\varepsilon}(t, x)=\delta_{0} * \beta^{\varepsilon}=\Delta E * \beta^{\varepsilon}=\int_{\mathbb{R}^{2}} \frac{x-y}{2 \pi|x-y|^{2}} \cdot \nabla_{x} \beta^{\varepsilon}(t, y) \mathrm{d} y
$$

(l'intégrale est en fait prise sur $B_{R}$ seulement). Ainsi, on se ramène à étudier

$$
\int_{0}^{T} \int_{\mathbb{R}^{2}} a^{\varepsilon} b^{\varepsilon} \varphi(t, x) \mathrm{d} x \mathrm{~d} t=\int_{0}^{T} \int_{B_{R}} \nabla_{x} \beta^{\varepsilon}(t, y) \cdot A^{\varepsilon}(t, y) \mathrm{d} y \mathrm{~d} t
$$

où

$$
A^{\varepsilon}(t, y)=\int_{B_{R}} a^{\varepsilon}(t, x) \frac{x-y}{2 \pi|x-y|^{2}} \mathrm{~d} x .
$$

Comme $\nabla \beta^{\varepsilon} \rightarrow \nabla(b \phi)$ dans $L^{2}\left((0, T) \times B_{R}\right)$, on est conduit à montrer que $A^{\varepsilon}$ est fortement compacte dans $L^{2}\left((0, T) \times B_{R}\right)$, ce qu'on justifie à l'aide d'arguments d'interpolation.

Pour appliquer ce résultat, on note que (9), combiné à la borne sur $J^{\varepsilon} \mathrm{du}$ Lemme 4 , assure que $\partial_{t} \rho^{\varepsilon}$ est bornée dans $L^{2}\left(0, T, W_{\text {loc }}^{-1,1}\left(\mathbb{R}^{2}\right)\right)$ et donc $\left(\int_{\mathbb{R}^{2}} \rho^{\varepsilon} \varphi \mathrm{d} x\right)_{\varepsilon>0}$ est compacte dans $C^{0}([0, T])$, pour tout $\varphi \in C_{0}^{\infty}\left(\mathbb{R}^{2}\right)$. On en déduit que $\rho^{\varepsilon}$ converge vers $\rho$ dans $C^{0}\left([0, T] ; L^{1}\left(\mathbb{R}^{2}\right)\right.$-faible $)$. En particulier cette dernière convergence permet de récupérer la condition initiale dans la limite $\varepsilon \rightarrow 0$. De même, on a convergence de $\widetilde{u^{\varepsilon}}$ vers $u$ dans $C^{0}\left([0, T] ; L^{2}\left(\mathbb{R}^{2}\right)\right.$-faible $)$. Finalement, on peut conclure avec l'énoncé suivant.

Théorème 1 On se place en dimension $N=2$. Les données initiales $f_{0}^{\varepsilon} \geq 0$, $f_{0}^{\varepsilon} \in L^{1}\left(\mathbb{R}^{2} \times \mathbb{R}^{2}\right)$ et $u_{0}^{\varepsilon} \in L^{2}\left(\mathbb{R}^{2}\right)$ vérifient $(5)$. Soit $0<T<\infty$. Alors, la densité macroscopique $\rho^{\varepsilon}$ converge dans $C^{0}\left([0, T] ; L^{1}\left(\mathbb{R}^{2}\right)\right.$-faible $)$ vers $\rho$, et $\widetilde{u}^{\varepsilon}=\varepsilon^{-1} u^{\varepsilon}$ converge faiblement dans $L^{2}\left(0, T ; H^{1}\left(\mathbb{R}^{2}\right)\right)$ et fortement dans $L_{\text {loc }}^{2}\left(\mathbb{R}^{+} \times \mathbb{R}^{2}\right)$ vers $\widetilde{u}$ où $(\rho, \widetilde{u})$ vérifient

$$
\left\{\begin{array}{l}
\partial_{t} \rho+\operatorname{div}_{x}(\widetilde{u} \rho-\nabla \rho)=0, \\
\partial_{t} \widetilde{u}+\operatorname{Div}_{x}(\widetilde{u} \otimes \widetilde{u})+\nabla_{x} p-\Delta_{x} \widetilde{u}=0, \\
\operatorname{div}_{x}(\widetilde{u})=0 .
\end{array}\right.
$$

Les données initiales sont données par

$$
\rho_{\mid t=0}=\rho_{0}, \quad \widetilde{u}_{\mid t=0}=\widetilde{u}_{0},
$$

où $\rho^{0}, \widetilde{u}^{0}$ sont les limites (faibles) de $\rho_{0}^{\varepsilon}, \widetilde{u}_{0}^{\varepsilon}$ respectivement. 


\section{Limite "hyperbolique"}

Un autre scaling intéressant consiste à poser dans (3)

$$
A=1=E, \quad B=\varepsilon^{-1}=D
$$

(i.e. le temps de relaxation est petit par rapport au temps d'observation, $\rho_{p}$ et $\rho_{g}$ sont du même ordre alors que le rayon des particules $a$ est petit par rapport à l'échelle d'observation). On s'intéresse donc à

$$
\left\{\begin{array}{l}
\partial_{t} f^{\varepsilon}+v \cdot \nabla_{x} f^{\varepsilon}+\frac{1}{\varepsilon} \nabla_{v} \cdot\left(\left(u^{\varepsilon}-v\right) f^{\varepsilon}-\nabla_{v} f^{\varepsilon}\right)=0 \\
\partial_{t} u^{\varepsilon}+\operatorname{Div}_{x}\left(u^{\varepsilon} \otimes u^{\varepsilon}\right)+\nabla_{x} p^{\varepsilon}-\Delta_{x} u^{\varepsilon}=\frac{1}{\varepsilon} \rho^{\varepsilon}\left(V^{\varepsilon}-u^{\varepsilon}\right)=\frac{1}{\varepsilon}\left(J^{\varepsilon}-\rho^{\varepsilon} u^{\varepsilon}\right), \\
\operatorname{div}_{x}\left(u^{\varepsilon}\right)=0, \\
\rho^{\varepsilon}(t, x)=\int f^{\varepsilon}(t, x, v) \mathrm{d} v, \quad \rho^{\varepsilon} V^{\varepsilon}(t, x)=\int v f^{\varepsilon}(t, x, v) \mathrm{d} v=J^{\varepsilon}(t, x) .
\end{array}\right.
$$

\subsection{Estimations a priori}

Des raisonnements analogues à ceux de la précédente section permettent d'établir les propriétés suivantes.

Proposition 3 Soit $\left(f^{\varepsilon}, u^{\varepsilon}\right)$ solution de (17) pour des donnnées vérifiant

$$
\left\{\begin{array}{l}
\int_{\mathbb{R}^{2}} \int_{\mathbb{R}^{2}} f_{0}^{\varepsilon}\left(1+x^{2}+v^{2}+\left|\ln \left(f_{0}^{\varepsilon}\right)\right|\right) \mathrm{d} v \mathrm{~d} x \leq C_{0}, \\
\int_{\mathbb{R}^{2}}\left|u_{0}^{\varepsilon}\right|^{2} \mathrm{~d} x \leq C_{0} .
\end{array}\right.
$$

Soit $0<T<\infty$. Alors, on a

i) $f^{\varepsilon}\left(1+x^{2}+v^{2}+\left|\ln \left(f^{\varepsilon}\right)\right|\right)$ est borné dans $L^{\infty}\left(0, T ; L^{1}\left(\mathbb{R}^{2} \times \mathbb{R}^{2}\right)\right)$,

ii) $u^{\varepsilon}$ est borné dans $L^{\infty}\left(0, T ; L^{2}\left(\mathbb{R}^{2}\right)\right)$ et dans $L^{2}\left(0, T ; H^{1}\left(\mathbb{R}^{2}\right)\right)$,

iii) $-\frac{1}{\sqrt{\varepsilon}} d^{\varepsilon}=\frac{1}{\sqrt{\varepsilon}}\left(\left(u^{\varepsilon}-v\right) \sqrt{f^{\varepsilon}}-2 \nabla_{v} \sqrt{f^{\varepsilon}}\right)$ est bornée dans $\left.L^{2}\left((0, T) \times \mathbb{R}^{2} \times \mathbb{R}^{2}\right)\right)$.

On en déduit des estimations pour les grandeurs macroscopiques.

Corollaire 1 On a

i) $\rho^{\varepsilon}\left(1+x^{2}+\left|\ln \left(\rho^{\varepsilon}\right)\right|\right)$ est borné dans $L^{\infty}\left(0, T ; L^{1}\left(\mathbb{R}^{2}\right)\right)$,

ii) $J^{\varepsilon}$ est borné dans $L^{\infty}\left(0, T ; L^{1}\left(\mathbb{R}^{2}\right)\right)$.

Avec les arguments de la Section 2, on remarque $\rho^{\varepsilon} u^{\varepsilon}$ est défini au moins dans $L_{\text {loc }}^{1}$. En fait, ici on peut même montrer que $J^{\varepsilon} \cdot u^{\varepsilon}$ et $\rho^{\varepsilon} u^{\varepsilon} \cdot u^{\varepsilon}$ sont bornés dans $L^{1}\left((0, T) \times \mathbb{R}^{2}\right)$. Enfin, on a une borne sur $\left|v-u^{\varepsilon}\right|^{2} f^{\varepsilon}$ dans $L^{1}\left((0, T) \times \mathbb{R}^{2} \times \mathbb{R}^{2}\right)$. En utilisant l'équation fluide, on s'aperçoit que $J^{\varepsilon}-\rho^{\varepsilon} u^{\varepsilon}$ tend vers 0 dans $\mathcal{D}^{\prime}\left((0, T) \times \mathbb{R}^{2}\right)$. En fait, on a une meilleure convergence. 
Lemme 6 La suite $J^{\varepsilon}-\rho^{\varepsilon} u^{\varepsilon}=\rho^{\varepsilon}\left(V^{\varepsilon}-u^{\varepsilon}\right)$ converge vers 0 dans $L^{2}\left(0, T ; L^{1}\left(\mathbb{R}^{2}\right)\right)$.

Cette propriété provient des propriétés de dissipation du système puisque

$$
\rho^{\varepsilon}\left|V^{\varepsilon}-u^{\varepsilon}\right|=\left|\int_{\mathbb{R}^{2}}\left(v-u^{\varepsilon}\right) f^{\varepsilon} \mathrm{d} v\right|=\left|\int_{\mathbb{R}^{2}} d^{\varepsilon} \sqrt{f^{\varepsilon}} \mathrm{d} v\right| .
$$

Il s'ensuit que

$$
\begin{aligned}
\int_{0}^{t}\left|\int_{\mathbb{R}^{2}} \rho^{\varepsilon}\left(V^{\varepsilon}-u^{\varepsilon}\right) \mathrm{d} x\right|^{2} \mathrm{~d} s & \\
\leq & \left(\sup _{0 \leq t \leq T} \int_{\mathbb{R}^{2}} \int_{\mathbb{R}^{2}} f^{\varepsilon} \mathrm{d} v \mathrm{~d} x\right) \times \int_{0}^{t} \int_{\mathbb{R}^{2}} \int_{\mathbb{R}^{2}}\left|d^{\varepsilon}\right|^{2} \mathrm{~d} v \mathrm{~d} x \mathrm{~d} s
\end{aligned}
$$

est $\mathcal{O}(\varepsilon)$

\subsection{Equations limites}

Les équations de moments s'écrivent ici

$$
\left\{\begin{array}{l}
\partial_{t} \rho^{\varepsilon}+\operatorname{div}_{x}\left(J^{\varepsilon}\right)=0 \\
\partial_{t} J^{\varepsilon}+\operatorname{Div}_{x}\left(\mathbb{P}^{\varepsilon}\right)=\frac{1}{\varepsilon}\left(\rho^{\varepsilon} u^{\varepsilon}-J^{\varepsilon}\right)
\end{array}\right.
$$

On rappelle que $\mathbb{P}^{\varepsilon}=\int_{\mathbb{R}^{2}} v \otimes v f^{\varepsilon} \mathrm{d} v$ est borné dans $L^{\infty}\left(0, T ; L^{1}\left(\mathbb{R}^{2}\right)\right)$ d'après la Proposition 3. Au moins pour une sous suite, on peut supposer que

$$
\begin{cases}\rho^{\varepsilon} \rightarrow \rho & \text { faiblement dans } L^{\infty}\left(0, T ; L^{1}\left(\mathbb{R}^{2}\right)\right), \\ u^{\varepsilon} \rightarrow u & \text { faiblement dans } L^{\infty}\left(0, T ; L^{2}\left(\mathbb{R}^{2}\right)\right), \\ \nabla_{x} u^{\varepsilon} \rightarrow \nabla_{x} u & \text { faiblement dans } L^{2}\left((0, T) \times \mathbb{R}^{2}\right),\end{cases}
$$

et $\partial_{t} \rho^{\varepsilon}$ est borné dans $L^{\infty}\left(0, T ; W^{-1,1}\left(\mathbb{R}^{2}\right)\right)$. D'après le lemme de produit, $\rho^{\varepsilon} u^{\varepsilon} \rightarrow \rho u$ au moins dans $\mathcal{D}^{\prime}\left((0, T) \times \mathbb{R}^{2}\right)$ et $J^{\varepsilon}=\rho^{\varepsilon} u^{\varepsilon}+\left(J^{\varepsilon}-\rho^{\varepsilon} u^{\varepsilon}\right) \rightarrow \rho u$, d'après le Lemme 6 . Alors, quand $\varepsilon \rightarrow 0$ la relation de conservation de la masse devient

$$
\partial_{t} \rho+\operatorname{div}_{x}(\rho u)=0,
$$

i.e. $\rho$ vérifie l'équation de transport avec vitesse $u$.

En exploitant l'équation fluide, l'équation de courant se réécrit

$$
\partial_{t}\left(u^{\varepsilon}+J^{\varepsilon}\right)+\operatorname{Div}_{x}\left(u^{\varepsilon} \otimes u^{\varepsilon}+\mathbb{P}^{\varepsilon}\right)+\nabla_{x} p^{\varepsilon}-\Delta u^{\varepsilon}=0 .
$$

Le terme de pression peut se décomposer sous la forme

$$
\begin{aligned}
\mathbb{P}^{\varepsilon}=\int_{\mathbb{R}^{2}}\left(\left(v-u^{\varepsilon}\right) \sqrt{f^{\varepsilon}}+2 \nabla_{v} \sqrt{f^{\varepsilon}}\right) \otimes v \sqrt{f^{\varepsilon}} \mathrm{d} v & \\
& +\int_{\mathbb{R}^{2}} u^{\varepsilon} \otimes v f^{\varepsilon} \mathrm{d} v-2 \int_{\mathbb{R}^{2}} \nabla_{v} \sqrt{f^{\varepsilon}} \otimes v \sqrt{f^{\varepsilon}} \mathrm{d} v .
\end{aligned}
$$


Le premier terme est $\mathcal{O}(\sqrt{\varepsilon})$ dans $L^{1}\left((0, T) \times \mathbb{R}^{2}\right)$; les second et troisième ne sont autres que $u^{\varepsilon} \otimes J^{\varepsilon}$ et $2 \rho^{\varepsilon}$ respectivement. On a donc

$$
\partial_{t}\left(u^{\varepsilon}+J^{\varepsilon}\right)+\operatorname{Div}_{x}\left(\left(u^{\varepsilon}+J^{\varepsilon}\right) \otimes u^{\varepsilon}\right)+\nabla_{x}\left(p^{\varepsilon}+2 \rho^{\varepsilon}\right)-\Delta u^{\varepsilon}=\operatorname{Div}_{x}\left(R^{\varepsilon}\right)
$$

avec $R^{\varepsilon} \rightarrow 0$ dans $L^{1}$. Sous réserve que le produit $\left(u^{\varepsilon}+J^{\varepsilon}\right) \otimes u^{\varepsilon}$ passe aussi à la limite, on obtient

$$
\partial_{t}((1+\rho) u)+\operatorname{Div}_{x}((1+\rho) u \otimes u)+\nabla_{x} \pi-\Delta u=0
$$

$\operatorname{avec} \operatorname{div}_{x}(u)=0$.

Il ne semble pas évident de justifier qu'une telle convergence a lieu. Une autre approche consiste à exploiter une méthode d'entropie modulée. L'idée est de comparer la suite $\left(\rho^{\varepsilon}, u^{\varepsilon}\right)$ avec $(\widetilde{\rho}, \Phi)$, solution régulière (au moins sur un court intervalle de temps $[0, T])$ du problème limite

$$
\left\{\begin{array}{l}
\partial_{t} \widetilde{\rho}+\operatorname{div}_{x}(\widetilde{\rho} \Phi)=0 \\
\partial_{t}((1+\widetilde{\rho}) \Phi)+\operatorname{Div}_{x}((1+\widetilde{\rho}) \Phi \otimes \Phi)+\nabla_{x} \Pi-\Delta \Phi=0 \\
\operatorname{div}_{x} \Phi=0
\end{array}\right.
$$

Pour des exemples où cette méthode a été utilisée avec succès, on consultera Brenier [2], ou Golse-Levermore-Saint-Raymond [11]. On introduit l'entropie relative

$$
\left\{\begin{array}{l}
\mathcal{H}\left(f^{\varepsilon}, u^{\varepsilon}\right)=H\left(f^{\varepsilon} \mid M_{\widetilde{\rho}, \Phi}\right)+\int_{\mathbb{R}^{2}}\left|u^{\varepsilon}-\Phi\right|^{2} \mathrm{~d} x \\
H\left(f^{\varepsilon} \mid M_{\widetilde{\rho}, \Phi}\right)=\int_{\mathbb{R}^{2}} \int_{\mathbb{R}^{2}}\left(f^{\varepsilon} \ln \left(\frac{f^{\varepsilon}}{M_{\widetilde{\rho}, \Phi}}\right)+M_{\widetilde{\rho}, \Phi}-f^{\varepsilon}\right) \mathrm{d} v \mathrm{~d} x .
\end{array}\right.
$$

La partie fluide $\int_{\mathbb{R}^{2}}\left|u^{\varepsilon}-\Phi\right|^{2} \mathrm{~d} x$ n'est autre que la norme $L^{2}$ entre $u^{\varepsilon}$ et $\Phi$. La partie cinétique $H\left(f^{\varepsilon} \mid M_{\widetilde{\rho}, \Phi}\right)$ est une mesure de la distance entre $f^{\varepsilon}$ et $M_{\widetilde{\rho}, \Phi}$. En particulier, on a

$$
H\left(f^{\varepsilon} \mid M_{\widetilde{\rho}, \Phi}\right)=\int_{\mathbb{R}^{2}} \int_{\mathbb{R}^{2}}\left(\frac{f^{\varepsilon}}{M_{\widetilde{\rho}, \Phi}} \ln \left(\frac{f^{\varepsilon}}{M_{\widetilde{\rho}, \Phi}}\right)-\frac{f^{\varepsilon}}{M_{\widetilde{\rho}, \Phi}}+1\right) M_{\widetilde{\rho}, \Phi} \mathrm{d} v \mathrm{~d} x \geq 0
$$

puisque $s \ln (s)-s+1 \geq 0$. Une suite de calculs un peu longue conduit à l'estimation suivante.

\section{Lemme 7 On a}

$$
\begin{aligned}
\mathcal{H}\left(f^{\varepsilon}, u^{\varepsilon}\right)(t) \leq C \mathcal{H}\left(f^{\varepsilon}, u^{\varepsilon}\right)(0)+C_{T} \int_{0}^{t} \mathcal{H}\left(f^{\varepsilon}, u^{\varepsilon}\right)(s) \mathrm{d} s+C_{T} \sqrt{\varepsilon} & \\
& +\int_{0}^{T} \int_{\mathbb{R}^{2}}\left(\rho^{\varepsilon}-\widetilde{\rho}\right)\left(u^{\varepsilon}-\Phi\right) \cdot G \mathrm{~d} x \mathrm{~d} t
\end{aligned}
$$

avec

$$
G=\frac{\nabla_{x} \Pi-\Delta \Phi}{1+\widetilde{\rho}}+\nabla_{x} \ln (\widetilde{\rho})
$$


On aimerait en conclure, avec une application du lemme de Gronwall, que $\mathcal{H}\left(f^{\varepsilon}, u^{\varepsilon}\right)(t)$ tend vers 0 quand $\varepsilon \rightarrow 0$, ce qui impliquerait $f^{\varepsilon} \rightarrow M_{\widetilde{\rho}, \Phi}, \rho^{\varepsilon} \rightarrow \widetilde{\rho}$ dans $L^{\infty}\left(0, T ; L^{1}\left(\mathbb{R}^{2}\right)\right)$ et $u^{\varepsilon} \rightarrow \Phi$ dans $L^{\infty}\left(0, T ; L^{2}\left(\mathbb{R}^{2}\right)\right)$. La difficulté est évidemment liée au terme $\iint\left(\rho^{\varepsilon}-\widetilde{\rho}\right)\left(u^{\varepsilon}-\Phi\right) \cdot G \mathrm{~d} x \mathrm{~d} t$. Soit $\widetilde{\rho}^{\varepsilon}$ l'unique solution (renormalisée et dans $\left.\mathcal{D}^{\prime}\right)$ dans $C^{0}\left([0, T] ; L^{2}\left(\mathbb{R}^{2}\right)\right)$ de l'équation de transport $\partial_{t} \widetilde{\rho}^{\varepsilon}+u^{\varepsilon} \cdot \nabla_{x} \widetilde{\rho}^{\varepsilon}=0$, associée à la donnée initiale $\rho_{0}^{\varepsilon}$, qu'on suppose régulière. On utilise ici les résultats de Di Perna-Lions [6]. On a

$$
\left\|\widetilde{\rho}-\widetilde{\rho}^{\varepsilon}\right\|_{L^{2}\left(\mathbb{R}^{2}\right)} \leq C_{T}\left(\left\|\widetilde{\rho}_{0}-\rho_{0}^{\varepsilon}\right\|_{L^{2}\left(\mathbb{R}^{2}\right)}+\left\|u^{\varepsilon}-\Phi\right\|_{L^{2}\left((0, T) \times \mathbb{R}^{2}\right)}\right)
$$

et on peut écrire

$$
\begin{array}{rl}
\mathcal{H}\left(f^{\varepsilon}, u^{\varepsilon}\right)(t) \leq C \mathcal{H}\left(f^{\varepsilon}, u^{\varepsilon}\right)(0)+C_{T} \int_{0}^{t} & \mathcal{H}\left(f^{\varepsilon}, u^{\varepsilon}\right)(s) \mathrm{d} s+C_{T} \sqrt{\varepsilon} \\
& +\int_{0}^{T} \int_{\mathbb{R}^{2}}\left(\rho^{\varepsilon}-\widetilde{\rho}^{\varepsilon}\right)\left(u^{\varepsilon}-\Phi\right) \cdot G \mathrm{~d} x \mathrm{~d} t .
\end{array}
$$

D'après les résulats de stabilité de [6], $\widetilde{\rho}^{\varepsilon}$ converge dans $C^{0}\left([0, T] ; L^{2}\left(\mathbb{R}^{2}\right)\right)$ vers $\bar{\rho}$ unique solution dans $C^{0}\left([0, T] ; L^{2}\left(\mathbb{R}^{2}\right)\right)$ de $\partial_{t} \bar{\rho}+u \cdot \nabla_{x} \bar{\rho}=0$. Or, d'après le lemme de produit, on a vu que $\rho^{\varepsilon}$ converge dans $C^{0}\left([0, T] ; L^{1}\left(\mathbb{R}^{2}\right)\right.$-faible) vers $\rho$, solution dans $\mathcal{D}^{\prime}$ de $\partial_{t} \rho+\operatorname{div}_{x}(\rho u)=0$. Il est extrêmement tentant d'en déduire que $\rho=\bar{\rho}$ et donc que

$$
\int_{0}^{T} \int_{\mathbb{R}^{2}}\left(\rho^{\varepsilon}-\widetilde{\rho}^{\varepsilon}\right)\left(u^{\varepsilon}-\Phi\right) \cdot G \mathrm{~d} x \mathrm{~d} t \underset{\varepsilon \rightarrow 0}{\longrightarrow} \int_{0}^{T} \int_{\mathbb{R}^{2}}(\rho-\bar{\rho})(u-\Phi) \cdot G \mathrm{~d} x \mathrm{~d} t=0 .
$$

Malheureusement, les propriétés dont on dispose sur $\rho$ (estimation $L^{1} \cap L \ln L$ ) et $u$ (estimation $H^{1}$ ) ne permettent pas d'appliquer les résultats d'unicité de [6] et à ce stade le résultat demeure formel.

\section{Références}

[1] Berthonnaud P. Limites fluides pour des modèles cinétiques de brouillards de gouttes monodispersés, C. R. Acad. Sci., 331 (2000) 651-654.

[2] Brenier, Y., Convergence of the Vlasov-Poisson system to the incompressible Euler equations, Comm. Partial Differential Equations, 25 (2000) 737-754.

[3] Caflisch R., Papanicolaou G., Dynamic theory of suspensions with Brownian effects, SIAM J. Appl. Math., 43 (1983) 885-906.

[4] Clouet J.F., Domelevo K., Solutions of a kinetic stochastic equation modeling a spray in a turbulent gas flow, Math. Models Methods Appl. Sci., 7 (1997) 239-263.

[5] Desvillettes L., About the modelling of complex flows by gas-particle methods, Preprint CMLA, 2001.

[6] DiPerna R., Lions P.-L., Ordinary differential equations, transport theory and Sobolev spaces, Invent. Math., 98 (1989) 511-547. 
[7] Domelevo K., Roquejoffre J. M., Existence and stability of traveling wave solutions in a kinetic model of two-phase flows, Comm. PDE, 24 (1999) 61-108.

[8] Domelevo K., Vignal M.-H., Limites visqueuses pour des systèmes de type Fokker-Planck-Burgers unidimensionnels, C. R. Acad. Sci., 332 (2001) 863-868.

[9] Dumas, Golse F., Homogenization of transport equations, SIAM J. Appl. Math., 60 (2000) 1447-1470.

[10] Gilbard, Trudinger N., Elliptic PDE of second order, (Springer, 1983).

[11] Golse F., Levermore C. D., Saint-Raymond L., La méthode de l'entropie relative pour les limites hydrodynamiques de modèles cinétiques Séminaire Equations aux Dérivées Partielles, 1999-2000, Exp. No. XIX, Ecole Polytechnique, Palaiseau, 2000.

[12] Goudon T., Asymptotic problems for a kinetic model of two-phase flow, Proc. Royal Soc. Edimburgh, 131, (2001) 1371-1384.

[13] Hamdache K., Global existence and large time behaviour of solutions for the Vlasov-Stokes equations, Japan J. Industrial and Appl. Math., 15 (1998) 51-74.

[14] Hamdache K., Umpublished work, Personal Communication.

[15] Herrero H., Lucquin-Desreux B., Perthame B., On the motion of dispersed balls in a potential flow : a kinetic description of the added mass effect, SIAM J. Appl. Math., 60 (1999) 61-83.

[16] Jabin P.E., Large time concentrations for solutions to kinetic equations with energy dissipation, Comm. PDE., 25 (2000) 541-557.

[17] JABIN P.E., Macroscopic limit of Vlasov type equations with friction, Ann. IHP Anal. Non Linéaire, 17 (2000) 651-672.

[18] Jabin P.E., Perthame B., Notes on mathematical problems on the dynamics of dispersed particles interacting through a fluid in Modeling in applied sciences, a kinetic theory approach, N. Bellomo, M. Pulvirenti Eds. (Birkhäuser, 2000), pp. 111-147.

[19] Lions P.L., Compactness in Boltzmann's equation via Fourier integral operators and applications. I, II, III, J. Math. Kyoto Univ. 34 (1994) 391-461, 1994 and 34 (1994) 539-584.

[20] Nieto J., Poupaud F., Soler J., High field limit for the VPFP system, Arch. Rat. Mech. Anal., 158 (2001) 29-59.

[21] Poupaud F., Soler J., Parabolic limit and stability of the Vlasov-PoissonFokker-Planck system, Math. Models Methods Appl. Sci., 10 (2000) 1027-1045.

[22] Russo G., Smereka P., Kinetic theory for bubbly flows I, II, SIAM J. Appl. Math., 56 (1996) 327-371.

[23] Simon J., Compact sets in $L^{p}(0, T ; B)$, Ann. Mat. Pura. Appl. IV, 146 (1987) 65-96.

[24] Williams F. A., Combustion theory (Benjamin Cummings Publ., 2nd ed., 1985). 
Labo. J.A. Dieudonné, UMR 6621

Université Nice-Sophia Antipolis, Parc Valrose

F-06108 NiCE CEDEX 02

goudon@math . unice.fr

www-math.unice.fr/ goudon

vasseur@math.unice.fr

DÉPARtement de Mathématiques et Applications, École Normale Supérieure,

45, RUE D'Ulm, F-75232 PARIS

jabin@dma.ens.fr 